\title{
One pot synthesis, characterization and mechanism of zinc glycinate monohydrate
}

\author{
HuiFeng ${ }^{1,2, a}$,Zhiwei Xu ${ }^{1}$, Qianfeng $\mathrm{Li}^{1}$, Xiuru Wang ${ }^{1}$ and Qiuyue Song ${ }^{1}$ \\ ${ }^{1}$ Heibei DonghuaJiheng Chemical Co. Ltd, Hengshui053000, China \\ 2School of Chemistry \& Chemical Engineering, Jiangsu University, Zhenjiang 212013, China \\ afenghui0827@163.com
}

Keywords:zinc glycinate monohydrate, glycine, zinc supplement,bioavailability.

\begin{abstract}
A facile method to control thesynthesis of zinc glycinate is described. $\mathrm{Zn}(\mathrm{Ac})_{2}$ and $\mathrm{ZnO}$ are used aszinc source, and $\mathrm{H}^{+}$is used as catalyst. The composition and structure of the chelate compounds are characterized by infrared spectroscopy(IR), thermogravimetric analysis(TGA) and $\mathrm{x}$-ray powder diffraction(XRD).It prove that the chelatecompoundsis monoclinic, and meanwhile we discusse the formation mechanism rationally.This is an effectiveand novelmethod for the synthesis ofhigh-yieldZn(Gly $)_{2} \bullet \mathrm{H}_{2} \mathrm{O}$ to realize industrial production.
\end{abstract}

\section{Introduction}

In recent years, how to properly use the trace mineral elements to improve its bioavailability is a very interesting but complex topic in the food nutritionand biochemistry. Particularly, Zinc deficiency has become a common phenomenon which will lead topoor immune system, low intelligence, loss of appetite and so on. As we all known, direct zinc supplement is not only bad absorption, but also will bring to negative effect[1-3]. Therefore, we need to find a dual role of zinc supplement nutritious and therapeutic to address the problem.

Compared with traditional zinc complement zinc sulfate $\left(\mathrm{ZnSO}_{4}\right)$ and zinc gluconate $\left(\mathrm{Zn}\left(\mathrm{C}_{6} \mathrm{H}_{11} \mathrm{O}_{7}\right)_{2}\right)$,zinc glycinate is a good candidate for it. The content of the zinc is $30.28 \%$ in the pure zinc glycinate. Up to now, it is a one of the highest zinc supplement reagent, many scholars at home and abroad have been study the synthesis and structure of $\mathrm{Zn}(\mathrm{Gly})_{2}$ at different levels[4-6]. Taken together, the synthesis of $\mathrm{Zn}(\mathrm{Gly})_{2}$ have aqueous synthesis method, solid-phase synthesis method, electrolytic method and phase equilibrium method [4,5]. The zinc source come from $\mathrm{Zn}, \mathrm{ZnCl}_{2}$, $\mathrm{ZnSO}_{4}$ and $\mathrm{ZnCO}_{3}$, etc. Although the analysis of structures of the zinc glycinate have been reported, there is not a systematic analysis and research. In this paper, we demonstrate the synthesis of $\mathrm{Zn}(\mathrm{Gly})_{2}$ via one pot chemistry method using $\mathrm{Zn}(\mathrm{Ac})_{2}$ and $\mathrm{ZnO}$ as zinc source. $\mathrm{Zn}(\mathrm{Ac})_{2}$ is used as promotion reagent of $\mathrm{ZnO}$ and catalyst to the formation of the $\mathrm{Zn}(\mathrm{Gly})_{2}$. The as-prepared crystal have been investigated by various characterization techniques and its formation mechanism is rationally discussed.

\section{Experimental section}

Sample Preparation.The reagents in this work, including zinc acetate dehydrate $\left(\mathrm{Zn}(\mathrm{Ac})_{2} \cdot 2 \mathrm{H}_{2} \mathrm{O}\right)$, zinc oxide $(\mathrm{ZnO})$, and ethanol(EtOH) were of analytical grade from the Tianjin Damao ChemicalFactory of China, the glycine and distilled water came from HeibeiDonghuaJiheng Chemical Co. Ltd. All chemicals were used as received without furtherpurification.

In a typical synthesis, $20 \mathrm{~g}$ glycine $(0.267 \mathrm{~mol}), 9.04 \mathrm{~g} \mathrm{ZnO}(0.111 \mathrm{~mol})$ and $4.88 \mathrm{~g} \mathrm{Zn}(\mathrm{Ac})_{2} \bullet 2 \mathrm{H}_{2} \mathrm{O}$ $(0.022 \mathrm{~mol})$ were added to distilled water $(60 \mathrm{ml})$ in a two-necked flask .Under stirring, it was heated to $90{ }^{\circ} \mathrm{C}$ in an oil bath and maintained at this temperature for $120 \mathrm{~min}$. Then the product were obtained by vacuum filtration, washed with ethanol several times, and dried at $30{ }^{\circ} \mathrm{C}$.

Characterization.Powder X-ray diffraction (XRD) patterns of the samples were collected on a D8 Advance diffractometer (Bruker, German) with graphite-monochromatized $\mathrm{Cu} \mathrm{K \alpha}$ radiation $(\lambda=$ 
1.54178 $\AA$ ). Infrared spectra were recorded on a Nicolet iS50 fourier transform spectrometer between 400 and $4000 \mathrm{~cm}^{-1}$. Thermogravimetric analysis(TGA) was carried out on a Netzsch STA 449C analyzer in the protection of $\mathrm{N}_{2}$ between 25 and $400{ }^{\circ} \mathrm{C}$ at a heating rate of $10{ }^{\circ} \mathrm{C} \mathrm{min}{ }^{-1}$.

\section{Results and discussion}

In the present work, we only use the $\mathrm{H}^{+}$as promoting solvent and catalyst, $\mathrm{ZnO}$ as zinc source in the synthesis process. However, the introduction of the trace $\mathrm{H}^{+}$fail in dissolving theundissovedZnO, and meanwhile the product is not easy to separation. Even if hot separation and crystallization, the yield of product is only about $20 \%$.In order to address the knotty problem, it is necessary to seek for a proper synthetic tactic. Very recently, our group developed a convenient strategy to synthesize $\mathrm{Zn}(\mathrm{Gly})_{2}$. The addition of $\mathrm{Zn}(\mathrm{Ac})_{2}$ can completely dissolve the $\mathrm{ZnO}$ at a certain condition, and it is easy to obtain the target products through separation at ambient temperature. Ifwe add the $\mathrm{Zn}(\mathrm{Ac})_{2}$ without $\mathrm{ZnO}$, the reaction result in forming lots of HAc so that the product can't be crystalized, precipitated and separated. In our synthesis strategy, the yield of the $\mathrm{Zn}(\mathrm{Gly})_{2}$ is $54.85 \%$ at first time, then adding the same amount of reactant to the first filtrated liquid to further reaction, we can get $98.92 \% \mathrm{Zn}(\mathrm{Gly})_{2}$. At the third time, the yield of the product is $98.72 \%$. We are believing that the reaction system has been designed by our group based on this idea(eqn $(1,2))$.

$$
\begin{aligned}
& \text { Gly }-\mathrm{H}+\mathrm{Zn}^{2+} \rightarrow \mathrm{Gly}-\mathrm{Zn}-\mathrm{Gly}+\mathrm{H}^{+}(1) \\
& \mathrm{H}^{+}+\mathrm{ZnO} \rightarrow \mathrm{Zn}^{2+}+\mathrm{H}_{2} \mathrm{O}
\end{aligned}
$$

Fig. 1 shows the X-ray diffraction (XRD) pattern of the Gly and as-prepared $\mathrm{Zn}(\mathrm{Gly})_{2}$. The diffraction peaks(Fig. 1a) at $2 \theta 14.9^{\circ}, 19.0^{\circ}, 23.9^{\circ}$ and $36.6^{\circ}$ match well with the characteristic peaks of monoclinic glycine ( $\alpha$-gly)[9,13].However, The main diffraction peaks (Fig.1b) of the zinc glycinate obviously occurr to displacement,and the interplanar spacing (d) and relative intensity are also changed. It indicates that the chelating effect of glycine and zinc, rather than the simple mixture of glycine and zinc. The diffraction peaks of the obtained zinc glycinate high and sharp indicate that the crystal structure is fine, and the zinc glycinate is confirmed as triclinic or pseudo-momoclinic[5].

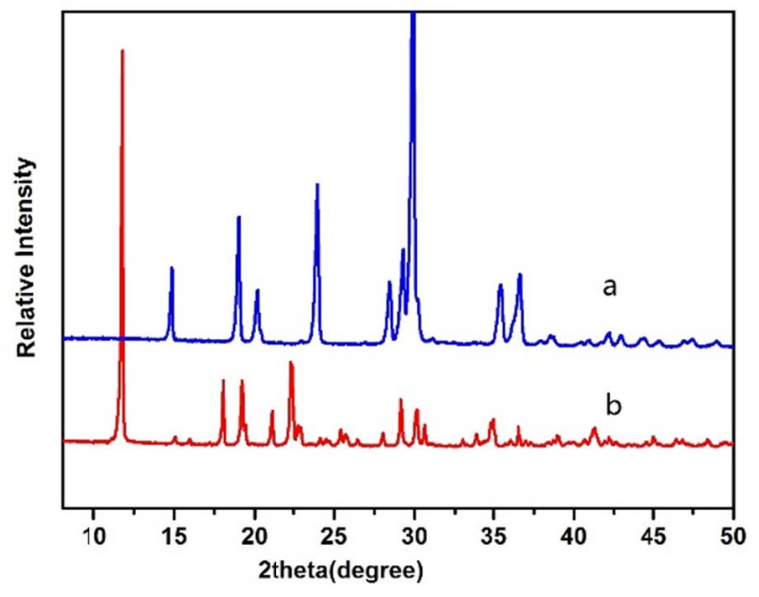

Fig. 1The XRD patterns of (a)the glycine and (b) zinc glycinate

Fourier Transform infrared spectroscopy (FTIR) is used to examine the product obtained from the typical synthetic procedure[10-12]. As shown in Fig.2a , the glycine have a broad absorption from $3100 \mathrm{~cm}^{-1}$ to $2400 \mathrm{~cm}^{-1}$, the weak band at about $3151 \mathrm{~cm}^{-1}$ is assigned to the antisymmetric stretching of the $\mathrm{NH}_{3}{ }^{+}$group. while the $\mathrm{Zn}(\mathrm{Gly})_{2}$ appear a sharp bands from $3400 \mathrm{~cm}^{-1}$ to $3100 \mathrm{~cm}^{-1}$, and the band of $\mathrm{NH}$ splits andshifts to higher frequencies at $3299.56 \mathrm{~cm}^{-1}$ and $3263.58 \mathrm{~cm}^{-1}$ after the formation of complexes, which are corresponding to the $v_{\mathrm{as}}$ and $v$ of $\mathrm{NH}$ bond, and meanwhilethe asymmetric 
bending and symmetric bending of $\mathrm{NH}_{3}{ }^{+}$have also changed.In the Fig. $2 \mathrm{~b}$, We can clearly see the stretching vibration peak of $\mathrm{Zn}-\mathrm{O}$ bond at $489 \mathrm{~cm}^{-1}[8]$, This further shows that the carboxyl group participated in coordination. Interestingly, we also observe stretching vibration peak of $\mathrm{Zn}-\mathrm{N}$ bond at about $598 \mathrm{~cm}^{-1}[7]$, and the peak of $\mathrm{O}-\mathrm{H}\left(1574.29 \mathrm{~cm}^{-1}\right)$ affords an accessorial proof for exsistence of crystal water.

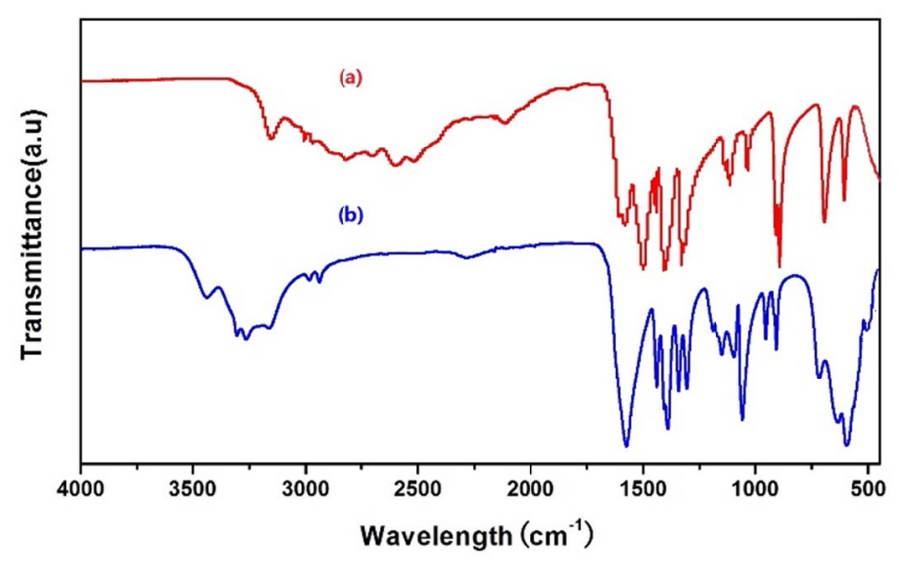

Fig. 2 The IR spectra of (a)the glycine and (b) zinc glycinate

TGA analyses areproperly performed to examine the content of crystall water.As displayed in Fig.3, the $\mathrm{Zn}(\mathrm{Gly})_{2}$ show an obvious weight loss in TGA heating process.7.75\% weight loss is measured for the as-prepared product.The weight loss can be attributed to the desorption of crystall water,and the weight loss date is consistent with the theoretical calculation value of only one crystall water, therefore the real structure is $\mathrm{Zn}(\mathrm{Gly})_{2} \cdot \mathrm{H}_{2} \mathrm{O}$.

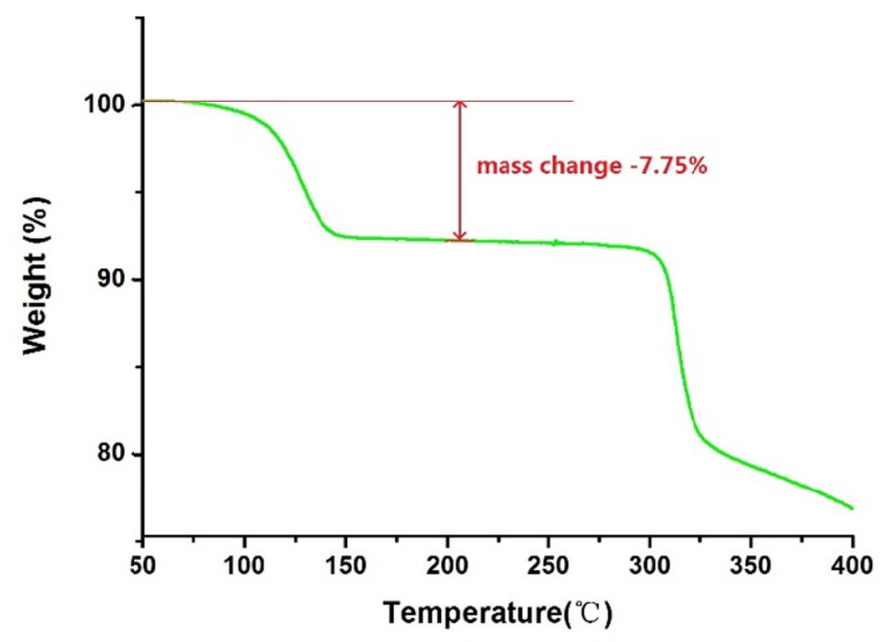

Fig. 3 The TG analysis of the Zn(Gly)2

\section{Conclusions}

In summary, The above investigations show that the route is an effective method for the synthesis of $\mathrm{Zn}(\mathrm{Gly})_{2} \cdot \mathrm{H}_{2} \mathrm{O}$ by one pot chemistry method using $\mathrm{Zn}(\mathrm{Ac})_{2}$ and $\mathrm{ZnO}$ as zinc source, and $\mathrm{H}^{+}$is used as catalyst, then discussthe formation mechanism rationaly. This work provides an alternative route 
for synthesizing metal chelates of glycine. We believe that this method is simple, inexpensive and easy to operation and will be helpful for industrial production.

\section{Acknowledgements.}

This work was financially supported by Hebei Donghua Jiheng Chemical Co. Ltd, thanks to Yongquan Yang for help in test.

\section{References}

[1] M. Yue, S. L. Fang, Z. Zhuo, D. D. Li, J. Feng,Zinc glycine chelate absorption characteristics in Sprague Dawley rat, J. Anim . Physiol. An. N.99(2015)457-464.

[2] D. Brugger, M .Buffler, W .Windisch,Development of an experimental model to assess the bioavailability of zinc in practical piglet diets, Arch. Anim .Nutr. 68(2014)423-424.

[3] S.J. Fairweather-Tait, T.E. Fox, S.G. Wharf,A preliminary study of the bio availability of iron and zincglycine chelates,Food. Addit. Contam. 9(1992)97-101.

[4] F. Rogalewicz, Y. Hoppilliard, G .Ohanessian,Structures and fragmentations of zinc (II) complexes of amino acids in the gas phase. III. Rearrangement versus desolvation in the electrospray formation of the glycine, Int. J. Mass .Spectrom. 206(2001)45-52.

[5]Barbara W. Low , F. L. Hirshfeld, F. M. Richards,Glycinate complexes of zinc and cadmium,J. Am. Chem. Soc.81(1959)4412-4416.

[6] J. A. Miceli , J. E. Stuehr,Kinetics of zinc-glycine interactions in aqueous solution, Inorg. Chem. 11 (1972)2763-2767.

[7] K. Krishnan, Robert .A. Plane,Raman study of glycine complexes of zinc (II), cadmium (II), and beryllium(II) and the formation of mixed complexes in aqueous solution,Inorg. Chem.6(1967)55-60.

[8]J .Das,Ivana .R. Evans ,Zinc glycolate: a precursor to ZnO. Inorg. Chem.48(2009)3508-3510.

[9] Z .Liu, L.Zhong, P .Ying, Z. Feng, C. Li,Crystallization of metastable $\beta$ glycine from gas phase via the sublimation of $\alpha$ or $\gamma$ form in vacuum,Biophys .Chem. 132(2008)18 - 22.

[10] E.S. Ferrari, R.J. Davey,Crystallization in polymorphic systems: the solution-mediated transformation of $\beta$ to $\alpha$ glycine, Cryst. Growth. Des. 3(2003)53 - 60 .

[11] M.T. Rosado, M.L.T.S. Duarte,R. Fausto,Vibrational spectra of acid and alkaline glycine salts, Vibr. Spectrosc. 16(1998)35 - 54.

[12] E. Ramachandran1, K. Baskaran, S. Natarajan,XRD, thermal, FTIR and SEM studies on gel grown $\gamma$ - glycine crystals, Cryst. Res. Technol. 42(2007)73- 77.

[13] E.S. Ferrari, R.J. Davey,Crystallization in polymorphic systems: the solution-mediated transformation of $\beta$ to $\alpha$ glycine, Cryst. Growth. Des. 3(2003)53 - 60. 